\title{
A generalization of a theorem of Minkowski
}

\author{
by \\ Marcin Mazur (Urbana, IL)
}

1. Introduction. Let $S$ be a ring. For an ideal $I$ of $S$, we denote by $R_{I}$ the natural map $\mathrm{GL}_{n}(S) \rightarrow \mathrm{GL}_{n}(S / I)$ and by $\mathrm{GL}_{n}(S, I)$ its kernel. For any subgroup $G$ of $\mathrm{GL}_{n}(S)$ we denote by $G(I)$ the kernel of $R_{I}$ restricted to $G$. The theorem of Minkowski mentioned in the title says that if $S$ is the ring of integers or the ring of 2-adic integers then any finite subgroup of $\mathrm{GL}_{n}(S, 2 S)$ is conjugated in $\mathrm{GL}_{n}(S)$ to diagonal matrices. For a local field or a number field $K$ we denote by $O_{K}$ the ring of integers of $K$. By $\mu_{p}$ we denote the group of roots of unity of $p$-power order. In the present note we prove the following generalization of Minkowski's result:

THEOREM 1. Suppose that either $K$ is a finite Galois extension of $\mathbb{Q}$ which is unramified at all finite primes except $p$ and such that there is a unique prime ideal $\gamma$ in $K$ over $p$ or $K$ is a finite Galois extension of $\mathbb{Q}_{p}$ contained in $\mathbb{Q}_{p}\left(\mu_{p} \infty\right)$ and $\gamma$ is the maximal ideal of $O_{K}$. Let $G \subseteq \operatorname{GL}_{n}\left(O_{K}\right)$ be a finite $\Gamma$-stable subgroup, where $\Gamma$ is the Galois group of $K$. Then $G(\gamma)$ can be conjugated to diagonal matrices by a matrix in $\mathrm{GL}_{n}(\mathbb{Z})\left(\right.$ in $\mathrm{GL}_{n}\left(\mathbb{Z}_{p}\right)$ in the local case).

The motivation behind this result came from our work on the following conjecture of Y. Kitaoka:

Conjecture 1. Any finite subgroup $G$ of $\mathrm{GL}_{n}(\overline{\mathbb{Z}})$ stable under the action of $\Gamma=\operatorname{Gal}(\overline{\mathbb{Q}} / \mathbb{Q})$ is pointwise fixed by the commutator of $\Gamma$.

Here $\overline{\mathbb{Z}}$ denotes the ring of all algebraic integers. Nevertheless, we think that Theorem 1 is of independent interest.

The innocent looking conjecture of Kitaoka has rather deep consequences in the theories of quadratic lattices, arithmetic groups and finite group schemes. For more information about this intriguing conjecture we refer the reader to [1], [3], [4], and [6].

2000 Mathematics Subject Classification: 20G30, 20G25, 11R33. 
Theorem 1 in the global case can be derived from the results of Kitaoka and Suzuki [2]. Their approach is quite different from ours and is based on the theory of quadratic lattices. In particular, it does not extend in any obvious way to the local situation. The present note provides in particular a new, more direct proof of the results of [2].

As explained in [4] and [5], Theorem 1 is in fact a statement about finite flat group schemes over $\mathbb{Z}$ (or $\mathbb{Z}_{p}$ ). Our technique can be interpreted as an alternative way of studying such group schemes.

2. Fundamental construction. In this section we recall the technical core of our approach, explained more carefully in [3].

Let $S$ be a commutative domain and suppose that $P$ is a finite abelian group sitting in $\mathrm{GL}_{n}(S)$ as a subgroup of diagonal matrices. In other words, there are abelian characters $\chi_{1}, \ldots, \chi_{n}$ of $P$ with values in the multiplicative group of $S$ such that $g=\operatorname{diag}\left(\chi_{1}(g), \ldots, \chi_{n}(g)\right)$ for all $g \in P$. After conjugating by a permutation matrix (which has entries in the prime subring of $S$ ) we can and will assume that there are integers $k_{0}=0<k_{1}<\ldots<$ $k_{s}<k_{s+1}=n$ such that $\chi_{i}=\chi_{j}$ iff $k_{t}<i, j \leq k_{t+1}$ for some $0 \leq t \leq s$. We say in this case that $P$ is in a strongly diagonal form. Set $N(P)$ for the normalizer of $P$ in $\mathrm{GL}_{n}(S)$ and $C(P)$ for its centralizer.

Lemma 1. The centralizer $C(P)$ of $P$ in $\mathrm{GL}_{n}(S)$ equals $\mathrm{GL}_{k_{1}-k_{0}}(S) \times$ $\ldots \times \mathrm{GL}_{k_{s+1}-k_{s}}(S)$. The group $W(P)=N(P) / C(P)$ is finite.

The first part of the lemma is pretty obvious and we omit the proof. The second part follows from the discussion below.

Note that $P$ is stable under the action of the automorphism group $\Gamma$ of $S$. Thus both $N(P)$ and $C(P)$ are $\Gamma$-stable. We denote by $\Pi_{n}$ the group of permutation matrices in $\mathrm{GL}_{n}(S)$. If $N \in N(P)$ and $\phi$ is the automorphism of $P$ induced by conjugation with $N$ then clearly the map $\chi \mapsto \chi \circ \phi$ permutes the characters $\chi_{1}, \ldots, \chi_{n}$. Let $\Sigma_{n}$ be the symmetric group of degree $n$. It is fairly obvious that we can choose a permutation $\pi \in \Sigma_{n}$ such that $\chi_{i} \circ \phi=\chi_{\pi(i)}$ and whenever $i<j$ and $\chi_{\pi(i)}=\chi_{\pi(j)}$ then $\pi(i)<\pi(j)$. Moreover, such a permutation is unique and if we denote by $T_{N}$ the corresponding permutation matrix then $N T_{N}^{-1} \in C(P)$ and $N \mapsto T_{N}$ is a group homomorphism $\sigma: N(P) \rightarrow \Pi_{n} \cap N(P)$. We denote by $\Pi_{P}$ the image of $\sigma$. Thus we get the following

Lemma 2. The group $N(P)$ is a semidirect product of $C(P)$ and $\Pi_{P}$. In particular, the induced action of $\Gamma$ on $W(P)$ is trivial.

Note that $N(P)$ acts by conjugation on $M_{k_{1}-k_{0}}(S) \times \ldots \times M_{k_{s+1}-k_{s}}(S)$, which has a basis consisting of matrices with one entry equal to 1 and all others being 0 . With respect to this basis the action of $N(P)$ defines a 
representation $\varrho$ of $N(P)$ in $\mathrm{GL}_{m_{1}^{2}+\ldots+m_{s+1}^{2}}(S)$, where $m_{i}=k_{i}-k_{i-1}$. It is clear that this representation respects the action of $\Gamma$. The kernel of $\varrho$ is equal to the product of the centers of $M_{k_{i}-k_{i-1}}(S)$. In particular, we get the following

Lemma 3. Suppose that $G$ is a finite, $\Gamma$-invariant subgroup of $N(P)$. Then $\varrho(G)$ is a finite, $\Gamma$-invariant subgroup of $\mathrm{GL}_{m_{1}^{2}+\ldots+m_{s+1}^{2}}(S)$ and the map $\varrho: G \rightarrow \varrho(G)$ commutes with the action of $\Gamma$. For any ideal $I$ of $S$ the map $\varrho$ takes $G(I)$ into $\varrho(G)(I)$.

We will use the above observations when $S$ is the ring of integers in a finite Galois extension of $\mathbb{Q}$ or $\mathbb{Q}_{p}$ and $\Gamma$ is the Galois group.

3. Main result. Let $K$ be an algebraic number field or a local field. Let $\beta$ be a prime ideal of the ring of integers $O_{K}$ of $K$ lying over a rational prime $p$. By $f$ we denote the ramification index of $\beta$.

The following is a variant of a well known Minkowski's lemma:

Minkowski's Lemma. Any torsion element of $\mathrm{GL}_{n}\left(O_{K}, \beta\right)$ has $p$ power order. If $\mathrm{GL}_{n}\left(O_{K}, \beta^{k}\right)$ contains an element of order $p^{s}$ then $k \leq$ $f p^{1-s} /(p-1)$.

For a proof see [3], Proposition 1.

LEMMA 4. Let $p$ be a prime. Suppose that $L \subseteq K$ are finite extensions of $\mathbb{Q}_{p}$ such that $K / L$ is Galois and the residue field of $K$ has $p$ elements. Denote by $\gamma$ the maximal ideal of $O_{K}$. Let $G \subseteq \mathrm{GL}_{n}\left(O_{K}\right)$ be a finite group stable under the action of the Galois group $\Gamma$ of $K / L$. Then for each $m \geq 1$ and any $\tau \in \Gamma, \tau$ acts on $G\left(\gamma^{m}\right) / G\left(\gamma^{m+1}\right)$ by raising to the $k$ th power, where $k$ is an integer such that $k \equiv\left(\pi^{m}\right)^{\tau} / \pi^{m}(\bmod \gamma)$ and $\pi$ is a generator of $\gamma$.

Proof. Let $\pi$ be a generator of $\gamma$. Fix $m>0$ and let $g, h \in G\left(\gamma^{m}\right)$. We can write $g=1+\pi^{m} a, h=1+\pi^{m} b$ for some $a, b \in M_{n}\left(O_{K}\right)$. Note that $g h \equiv 1+\pi^{m}(a+b)\left(\bmod \pi^{m+1}\right)$. This shows that the map $R$ which assigns to each $g \in G\left(\gamma^{m}\right)$ the reduction $\bmod \gamma$ of $(g-I) / \pi^{m}$ is a group homomorphism $R: G\left(\gamma^{m}\right) \rightarrow M_{n}\left(O_{K} / \gamma\right)$. The kernel of this homomorphism equals $G\left(\gamma^{m+1}\right)$. Let $\tau \in \Gamma$. By our assumption $O_{K} / \gamma$ has $p$ elements. In particular, there is an integer $k$ such that $k \equiv\left(\pi^{m}\right)^{\tau} / \pi^{m}(\bmod \gamma)$. Thus $R\left(g^{\tau}\right)=k R(g)$ for all $g \in G\left(\gamma^{m}\right)$. Consequently, $g^{\tau} g^{-k} \in G\left(\gamma^{m+1}\right)$. It follows that $\tau$ acts on $G\left(\gamma^{m}\right) / G\left(\gamma^{m+1}\right)$ by raising to the $k$ th power.

Suppose now that $K$ is a finite Galois extension of $\mathbb{Q}$ or $\mathbb{Q}_{p}$ with Galois group $\Gamma$. Let $G \subseteq \mathrm{GL}_{n}\left(O_{K}\right)$ be a finite $\Gamma$-stable group. Recall that for a prime $\beta$ of $O_{K}$ we defined $G(\beta)$ as the kernel of $R_{\beta}($ reduction $\bmod \beta$ ) restricted to $G$. Let $p$ be the rational prime in $\beta$. Thus $G(\beta)$ is a normal 
$p$-subgroup of $G$ by Minkowski's Lemma. In the global case, define $G(p)$ to be the subgroup of $G$ generated by the elements of all the groups $G(\beta)$ with $\beta$ containing $p$. Clearly $G(p)$ is a normal $p$-subgroup of $G$ stable under the action of $\Gamma$. By $D(\beta), I(\beta)$ we denote the decomposition and inertia subgroups of $\beta$ respectively.

Lemma 5. Let $K / \mathbb{Q}$ be a finite Galois extension with Galois group $\Gamma$ and let $G$ be a finite $\Gamma$-stable subgroup of $\mathrm{GL}_{n}\left(O_{K}\right)$. If $\beta, \beta^{\prime}$ are primes of $O_{K}$ lying over distinct rational primes $p, p^{\prime}$ then $I\left(\beta^{\prime}\right)$ acts trivially on $G(\beta)$.

Proof. Since $G(p)$ is a $p$-group and $G\left(\beta^{\prime}\right)$ is a $p^{\prime}$-group, it follows that $G(p) \cap G\left(\beta^{\prime}\right)=1$. In particular, $R_{\beta^{\prime}}$ is injective on $G(p)$ so the action of $I\left(\beta^{\prime}\right)$ on $G(p)$ is trivial. Since $G(\beta)$ is contained in $G(p)$, the lemma follows.

Proof of Theorem 1. Suppose that the theorem is false. Let $b$ be the order of a counterexample of minimal possible order (when both $n$ and $K$ vary) and let $n$ be minimal such that there is a counterexample $G$ of order $b$ in $\mathrm{GL}_{n}\left(O_{K}\right)$ for some $K$. We can assume that $\Gamma$ acts faithfully on $G$. Since $\gamma$ is the unique prime over $p$, we deduce that $G(\gamma)$ is $\Gamma$-stable. Thus $G=G(\gamma)$ by minimality of $G$. By Minkowski's Lemma the group $G$ is a $p$-group. Let $F$ be the Frattini subgroup of $G$. Then $F$ is a proper characteristic subgroup of $G$, so it is $\Gamma$-stable. Let $H$ be a maximal proper $\Gamma$-stable subgroup of $G$ containing $F$. By minimality of $G$ we can assume that $H$ consists of diagonal matrices and is in a strongly diagonal form (after conjugation by an element in $\mathrm{GL}_{n}(\mathbb{Z})$ (or $\mathrm{GL}_{n}\left(\mathbb{Z}_{p}\right)$ ) if necessary).

Lemma 6. The group $H$ is central in $\mathrm{GL}_{n}(K)$, i.e. consists of scalar matrices.

Proof. The group $G$ is a subgroup of the normalizer of $H$ in $\mathrm{GL}_{n}$. Thus we can use the results of Section 2. In particular, any $g \in G$ can be written as $c w$, where $w$ is a permutation matrix in the subgroup $\Pi_{H}$ of the normalizer of $H$ and $c$ centralizes $H$, i.e. $c w=g \in \mathrm{GL}_{n}\left(O_{K}\right)$. By reducing $\bmod \gamma$ we find that $R_{\gamma}(c)=R_{\gamma}(w)^{-1}$ in $\operatorname{GL}_{n}\left(O_{K} / \gamma\right)$. Recall that the centralizer of $H$ consists of block-diagonal matrices. Thus $R_{\gamma}(w)=R_{\gamma}(c)^{-1}$ is blockdiagonal. Since $w$ is a permutation matrix, it is block-diagonal and therefore centralizes $H$. Consequently, $w=I$ by the very definition of $\Pi_{H}$. The upshot is that $G$ centralizes $H$.

If $H$ is not central in $\mathrm{GL}_{n}$ then the centralizer of $H$ is a product of $\mathrm{GL}_{m}$ 's of dimensions smaller than $n$. Clearly, the image of $G$ in at least one of these $\mathrm{GL}_{m}$ 's is a counterexample to Theorem 1, which contradicts the minimality of $n$. Thus $H$ is central in $\mathrm{GL}_{n}$. 
If the field $K$ contains $p$ th roots of unity then for any $\gamma \in \Gamma$ we denote by $i(\gamma)$ the smallest positive integer such that $\gamma$ acts on the $p$ th roots of 1 by raising to the power $i(\gamma)$. We call $i$ the cyclotomic character of $\Gamma$.

\section{LEMma 7. The group $H$ is trivial.}

Proof. Suppose that $H$ is not trivial. Then $K$ contains $p$ th roots of 1 . Associated with $H$ is the representation $\varrho$ discussed in Section 2. The image $\varrho(G) \in \mathrm{GL}_{n^{2}}\left(O_{K}\right)$ is isomorphic to $G / H$. By Lemma 3 we know that $\varrho(G)$ is a finite $\Gamma$-stable subgroup of $\mathrm{GL}_{n^{2}}\left(O_{K}, \gamma\right)$. Moreover, the action of $\Gamma$ on $\varrho(G)=G / H$ is the same as the induced action from $G$. By minimality of our counterexample we conclude that $\varrho(G)$ can be conjugated to diagonal matrices by an element of $\mathrm{GL}_{n^{2}}(\mathbb{Z})$ (or $\mathrm{GL}_{n^{2}}\left(\mathbb{Z}_{p}\right)$ ). In particular, $\Gamma$ acts on $G / H$ via the cyclotomic character $i$ (i.e. the action of $\tau$ on $G / H$ is by raising to a power $i(\tau))$ and therefore every subgroup of $G / H$ is $\Gamma$-stable. Consequently, $G / H$ is cyclic of order $p$ (recall that $H$ is maximal among proper $\Gamma$-stable subgroups of $G$ containing the Frattini subgroup). Since $H$ is central, $G$ is abelian. Let $g \in G$ be such that its image in $G / H$ is a generator (i.e. $g \notin H$ ). For $\tau \in \Gamma$ there is an integer $i$ such that $k^{\tau}=k^{i}$ for any $k \in H$. Clearly $p \mid i(\tau)-i$. Thus $g^{\tau}=g^{i} h$ for some $h \in H$ (note that the action of $\tau$ on $G / H$ is by raising to power $i$ ). By raising both sides of the last equality to $p$ th power and using the fact that $g^{p} \in H$ we conclude that $h^{p}=1$.

Suppose that $g^{p} \neq 1$. Then $g^{p}$ generates a nontrivial subgroup $C$ of $H$ and therefore $h \in C$ (recall that $H$ is cyclic and $h^{p}=1$ ). Thus the subgroup generated by $g$ is $\Gamma$-stable and therefore equals $G$ by minimality of our counterexample. Let $g^{p}=\xi I$ for some root of unity $\xi$. Pick $u \in L=K\left(\xi^{1 / p}\right)$ such that $u^{p}=\xi^{-1}$. Note that the field $L$ satisfies all assumptions of Theorem 1. In the local case this is obvious. In the global case, the inertia $I$ of any prime of $L$ over $p$ surjects onto the Galois group of $K / \mathbb{Q}$ (which coincides with the inertia subgroup of the unique prime of $K$ over $p)$, i.e. $\operatorname{Gal}(L / K) I=\operatorname{Gal}(L / \mathbb{Q})$. Since $\operatorname{Gal}(\mathbb{Q}(u) / \mathbb{Q})$ is abelian, elements of $\operatorname{Gal}(L / K)$ are central in $\operatorname{Gal}(L / \mathbb{Q})$ and therefore $I$ is normal in $\operatorname{Gal}(L / \mathbb{Q})$. Since $\mathbb{Q}$ has no unramified extensions, it follows that $I=\operatorname{Gal}(L / \mathbb{Q})$ and there is a unique prime over $p$ in $L$.

Let $\tau$ be an automorphism of $L$. There is an integer $t$ such that $g^{\tau}=g^{t}$. Also, $u^{\tau}=u^{s}$ for some integer $s$. Clearly we have $u^{p s}=u^{p t}$ so that $u^{t-s}$ is a $p$ th root of 1 . Now consider the element $q=u g$. Plainly, $q^{p}=1$. Also, $q^{\tau}=u^{s} g^{t}=w q^{t}$ for some $p$ th root of unity $w$. Thus, the subgroup generated by $q$ and elements $w I, w$ a $p$ th root of 1 , is $\Gamma$-stable and it is an elementary abelian $p$-group of order $p^{2}$. Note that $g$ is conjugated to diagonal matrices by an element of $\mathrm{GL}_{n}(\mathbb{Z})$ (or $\mathrm{GL}_{n}\left(\mathbb{Z}_{p}\right)$ ) iff $q$ is such. Thus we can assume that $G$ is elementary abelian of order $p^{2}$ and $H$ is cyclic of order $p$. 
Suppose now that $g^{p}=1$. Then elements of order $p$ in $H$ and $g$ generate an elementary abelian $p$-group of order $p^{2}$ which is $\Gamma$-stable and it has to equal $G$ by minimality of our counterexample.

In any case, we can assume that $G$ is elementary abelian of order $p^{2}$. For any $\tau \in \Gamma$ we have $g^{\tau}=g^{i(\tau)} h(\tau)$, where $i$ is the cyclotomic character and $h(\tau) \in H$. An easy calculation shows that $g^{\tau_{1} \tau_{2}}=g^{i\left(\tau_{1}\right) i\left(\tau_{2}\right)} h\left(\tau_{1}\right)^{i\left(\tau_{2}\right)} h\left(\tau_{2}\right)^{i\left(\tau_{1}\right)}$. Consequently, $\tau_{1} \tau_{2}$ and $\tau_{2} \tau_{1}$ act in the same way on $G$. Thus, we can assume that $\Gamma$ is abelian.

If $p$ is odd this implies that $\Gamma$ is cyclic. Let $\tau$ be a generator of $\Gamma$. Note that $g^{\tau}=g^{i(\tau)} h$ for some $h \in H$. An easy calculation shows that $g^{\tau^{p-1}}=$ $g h^{(p-1) i(\tau)^{p-2}}=g h_{1}$. If $h \neq 1$ then $\tau$ has order $p(p-1)$ and $h_{1}=\xi I$ for a primitive $p$ th root of unity $\xi$. It follows that $K$ is the cyclotomic extension of $\mathbb{Q}$ of degree $p(p-1)$ (we use Kronecker-Weber theorem in the global case; recall that $p$ is the only ramified prime in $K$ ). Thus there is a primitive $p^{2}$ th root of unity $\zeta$ in $K$ such that $\zeta^{\tau^{p-1}}=\zeta \xi^{-1}$. Note that $(g \zeta)^{\tau^{p-1}}=g \zeta$, i.e. $g \zeta$ has entries in $\mathbb{Q}_{p}\left(\xi_{p}\right)$ (we pass to the completion at $p$ in the global case). Thus $g \zeta$ is an element of order $p^{2}$ in the congruence subgroup $\mathrm{GL}_{n}\left(\mathbb{Z}_{p}\left[\xi_{p}\right],\left(1-\xi_{p}\right)\right)$, which contradicts the inequality in Minkowski's Lemma. Thus $h=1$ and therefore the subgroup generated by $g$ is $\Gamma$-stable. This contradicts the fact that $G$ is a minimal counterexample and $H$ is nontrivial.

If $p=2$ then $\Gamma$ acts trivially on $H$, so we can assume that no nontrivial element of $\Gamma$ fixes $g$. In other words, $\Gamma$ is cyclic of order 2 and $g^{\tau}=-g$ for a generator $\tau$ of $\Gamma$. Note that in this case $K$ equals $\mathbb{Q}[w]$, where $w$ is one of $i, \sqrt{2}, \sqrt{-2}$. In any case, $w^{\tau}=-w$ and therefore $w g \in \mathrm{GL}_{n}\left(\mathbb{Q}_{2}\right)$ (we pass to the completion at 2 in the global case). If $w=i$, we find that $w g \in$ $\mathrm{GL}_{n}\left(\mathbb{Z}_{2}, 2\right)$ has order 4 , which contradicts Minkowski's Lemma. Otherwise, $w g$ has integral entries with nontrivial 2-adic valuation, so $w g=2 A$ where $A$ has integral entries. But then $(w g)^{2}= \pm 2 I=4 A^{2}$, which is a contradiction again. All this shows that $H$ has to be trivial.

By Lemma 7, $G$ is elementary abelian with no nontrivial proper $\Gamma$-stable subgroups. For every $r \geq 1$ the congruence subgroups $G\left(\gamma^{r}\right)$ are $\Gamma$-stable. Thus there is $r>0$ such that $G=G\left(\gamma^{r}\right)$ and $G\left(\gamma^{r+1}\right)=1$. Observe that the residue field $O_{K} / \gamma$ has $p$ elements. In fact, this is clear in the local case. In the global case, since $\gamma$ is the only prime over $p$ in $K / \mathbb{Q}$, we infer that $\Gamma=D(\gamma)$ is the decomposition group of $\gamma$. Thus, the inertia subgroup $I(\gamma)$ is normal in $\Gamma$ and its fixed field is an extensions of $\mathbb{Q}$ unramified at all finite primes. Consequently, $\Gamma=I(\gamma)$ and $O_{K} / \gamma$ has $p$ elements. Note that $\Gamma$ is the Galois group of the local extension $K_{\gamma} / \mathbb{Q}_{p}$. By Lemma $4, \Gamma$ preserves all subgroups of $G$. It follows that $G$ is cyclic of order $p$. Therefore we can assume that $\Gamma$ is cyclic of order $p-1$ and $K=\mathbb{Q}\left(\xi_{p}\right)\left(K=\mathbb{Q}_{p}\left(\xi_{p}\right)\right.$ in the local case). From the inequality in Minkowski's Lemma we see that $r=1$. 
Note that $\pi=1-\xi_{p}$ is a generator of $\gamma$ and $\pi^{\tau} / \pi=i(\tau)(\bmod \pi)$. Thus $\Gamma$ acts on $G$ via the cyclotomic character by Lemma 4 .

Let $g$ be a generator of $G$. For every $p$ th root of unit $\xi$ (including $\xi=1$ ) define $A_{\xi}=\left(\sum_{j=0}^{p-1} \xi^{j} g^{j}\right) / p$. Since the action on both $\xi$ and $g$ is via the cyclotomic character, we find that $A_{\xi}$ is fixed by $\Gamma$, i.e. has entries in $\mathbb{Q}$ $\left(\mathbb{Q}_{p}\right.$ in the local case). Note that $A_{\xi}=(\pi / p) \sum_{j=0}^{p-1} \xi^{j} b_{j}$, where $\pi=1-\xi_{p}$ and $b_{j}=\left(g^{j}-I\right) / \pi \in M_{n}\left(O_{K}\right)$. In particular, each entry of $A_{\xi}$ has $p$-adic valuation at least $1 /(p-1)-1>-1$. But these entries are in $\mathbb{Q}\left(\mathbb{Q}_{p}\right)$, so they are in fact in $\mathbb{Z}\left(\right.$ or $\left.\mathbb{Z}_{p}\right)$, i.e. $A_{\xi} \in M_{n}(\mathbb{Z})\left(A_{\xi} \in M_{n}\left(\mathbb{Z}_{p}\right)\right.$ in the local case). Note now that $A_{\xi}$ are commuting idempotent matrices and their sum equals the identity matrix. Also, $g A_{\xi}=\xi A_{\xi}$. Let $e_{i}$ be the standard basis of $K^{n}$. The elements $A_{\xi} e_{i}$ are eigenvectors for $g$ and they generate $\mathbb{Z}^{n}$ $\left(\right.$ resp. $\left.\mathbb{Z}_{p}^{n}\right)$. We can chose among them a basis of $\mathbb{Z}^{n}$ (resp. $\mathbb{Z}_{p}^{n}$ ), which shows that $g$ can be conjugated to diagonal matrix by an element of $\mathrm{GL}_{n}(\mathbb{Z})$ $\left(\mathrm{GL}_{n}\left(\mathbb{Z}_{p}\right)\right.$ in the local case). This contradicts our assumption that $G$ is a counterexample. The proof of Theorem 1 is now complete.

Question. Let $K$ be a finite Galois extension of $\mathbb{Q}_{p}$ which is totally ramified and set $\gamma$ for the maximal ideal of $O_{K}$. Suppose that $G$ is a finite subgroup of $\mathrm{GL}_{n}\left(O_{K}, \gamma\right)$ stable under the action of the Galois group $\Gamma$ of $K / \mathbb{Q}_{p}$. Is it true that $G$ can be conjugated to diagonal matrices by an element of $\mathrm{GL}_{n}\left(\mathbb{Z}_{p}\right)$ ?

Most of the proof of Theorem 1 works in the local situation, but the problem is that not all totally ramified extensions of $\mathbb{Q}_{p}$ are contained in $\mathbb{Q}_{p}\left(\mu_{p^{\infty}}\right)$. An affirmative answer to our question would be very useful for attacking Conjecture 1.

4. Consequences. Let $K / \mathbb{Q}$ be a finite Galois extension and write $O_{K}$ for the ring of integers in $K$. Consider a finite subgroup of $\mathrm{GL}_{n}\left(O_{K}\right)$ stable under the action of $\Gamma=\operatorname{Gal}(K / \mathbb{Q})$. The group $G$ acts in a natural way on $\mathbb{Z}^{n} \otimes O_{K}\left(\left(a_{i, j}\right) e_{i}=\sum a_{i, j} e_{j}\right)$. After Y. Kitaoka, we say that $G$ is of A-type if there exists a decomposition $\mathbb{Z}^{n}=\bigoplus_{i=1}^{k} M_{i}$ such that for every $g \in G$ there are a permutation $\pi(g)$ of $\{1, \ldots, k\}$ and roots of unity $\varepsilon_{i}(g)$ such that $\varepsilon_{i}(g) g M_{i}=M_{\pi(g)}$ for $i=1, \ldots, k$.

It is clear that groups of $A$-type are contained in $\mathrm{GL}_{n}\left(K^{\mathrm{ab}}\right)$ (recall that $K^{\mathrm{ab}}$ is the maximal abelian subextension of $K / \mathbb{Q}$ ). But being of $A$-type means much more. It is not hard to see that if $G$ has odd order then it is of $A$-type iff it is conjugated by an element of $\mathrm{GL}_{n}(\mathbb{Z})$ to a semidirect product $D B$ where $D$ is normal in $G$ and consists of diagonal matrices and $B \subseteq \mathrm{GL}_{n}(\mathbb{Z})$. When the order of $G$ is even, the group theoretic properties are a little harder to spell out. In this case, $G$ can be conjugated over $\mathbb{Z}$ to a subgroup of a semidirect product $D B$ as above. 
The following theorem was proved by Kitaoka and Suzuki [2]:

Theorem 2. If $\Gamma=\operatorname{Gal}(K / \mathbb{Q})$ is nilpotent then any finite $\Gamma$-stable subgroup of $\mathrm{GL}_{n}\left(O_{K}\right)$ is of A-type.

Below we give a new proof of this result, based on Theorem 1 and the methods of Section 2. For this we need a few lemmas.

Lemma 8. Let $K$ be a Galois extension of $\mathbb{Q}$. Suppose that $A \in \mathrm{GL}_{n}\left(O_{K}\right)$ has the property that for any $\tau \in \operatorname{Gal}(K / \mathbb{Q})$ there is a diagonal matrix $D_{\tau}=\operatorname{diag}\left(\xi_{1}(\tau), \ldots, \xi_{n}(\tau)\right)$ such that $\xi_{i}(\tau)$ 's are roots of unity and $A^{\tau}=$ $D_{\tau} A$. Then $A=D M$ where $D$ is a diagonal matrix of finite order and $M \in \mathrm{GL}_{n}(\mathbb{Z})$.

Proof. Let $A=\left(a_{i, j}\right)$. For every $i$ there exists $k$ such that $a_{i}=a_{i, k} \neq 0$. We have $a_{i, j}^{\tau}=\xi_{i}(\tau) a_{i, j}$ for all $j$. In particular, $a_{i}^{-1} a_{i, j}$ is stable under the action of $\operatorname{Gal}(K / \mathbb{Q})$ for all $j$. Thus there are rational numbers $q_{i, j}$ such that $a_{i, j}=a_{i} q_{i, j}$. For a given $i$ there is a rational number $q_{i}$ such that $m_{i, j}=q_{i} q_{i, j}$ are integers with no common factor. Set $d_{i}=q_{i}^{-1} a_{i}$ and let $D$ be the diagonal matrix with $d_{1}, \ldots, d_{n}$ on the diagonal. Clearly $A=D M$, where $M=\left(m_{i, j}\right)$. Since each row of $M$ consists of integers with no common factor and $A$ has entries in $O_{K}$, it follows that $d_{i}$ is an algebraic integer for all $i$. Moreover, $\operatorname{det} A=d_{1} \ldots d_{n} \operatorname{det} M$ is a unit in $O_{K}$, which implies that each $d_{i}$ is a unit and $M \in \mathrm{GL}_{n}(\mathbb{Z})$. Let $N$ be a natural number such that $\xi_{i}(\tau)^{N}=1$ for all automorphisms $\tau$ and all $i$. Since $d_{i}^{\tau}=\xi_{i}(\tau) d_{i}$, we deduce that $\left(d_{i}^{N}\right)^{\tau}=d_{i}^{N}$ for all $\tau \in \operatorname{Gal}(K / \mathbb{Q})$. Thus $d_{i}^{N} \in \mathbb{Q}$. Since the only units in $\mathbb{Q}$ are 1 and -1 we conclude that $d_{i}$ is a root of unity.

LEMmA 9. Let $K$ be a number field which has no abelian extensions unramified at all finite primes. Let $\beta$ be a prime of $O_{K}$ and $L$ a Galois extension of $K$ with nilpotent Galois group which is unramified at all finite primes different from $\beta$. Then there is a unique prime in $O_{L}$ over $\beta$ which is totally ramified.

Proof. We use induction on the order of the Galois group $\Gamma$ of $L / K$. Let $\pi$ be a prime of $O_{L}$ over $\beta$. If $\Gamma$ is abelian and the inertia group $I(\pi)$ is a proper subgroup of $\Gamma$ then $L^{I(\pi)}$ is a nontrivial abelian extension of $K$, unramified at all finite primes, which contradicts our assumption about $K$. Thus $\Gamma=I(\pi)$ and consequently $\pi$ is the only prime in $O_{L}$ over $\beta$.

In general, let $F$ be the Frattini subgroup of $\Gamma$. In particular, $F$ is a normal subgroup and $\Gamma / F$ is abelian. Thus $L^{F}$ is an abelian extension of $K$ so there is a unique prime $\gamma$ of $L^{F}$ over $\beta$. In other words, $\Gamma / F=I(\gamma)$. Note that under the natural projection $\Gamma \rightarrow \Gamma / F$ the inertia group $I(\pi)$ is mapped onto $I(\gamma)$. Thus $\Gamma=I(\pi) F$. Directly from the definition of the Frattini subgroup it follows that $I(\pi)=\Gamma$, which is exactly what we want to show. 
Now we are in a position to prove Theorem 2. Let then $G$ be a finite $\Gamma$-stable subgroup of $\mathrm{GL}_{n}\left(O_{K}\right)$, where $K / \mathbb{Q}$ is a finite Galois extension with nilpotent Galois group $\Gamma$. Fix a rational prime $p$. Let $I$ be the subgroup of $\Gamma$ generated by all the inertia subgroups $I(\beta)$ such that $\beta$ does not contain $p$. For any prime $\gamma$ of $K$ over $p$ the group $I$ acts trivially on $G(\gamma)$ by Lemma 5 . Recall that $G(p)$ is the subgroup of $G$ generated by all the $G(\gamma)$ with $\gamma$ over $p$. Thus $G(p)$ is a normal $\Gamma$-stable $p$-subgroup of $G$ and $I$ acts trivially on it. The fixed field $K^{I}$ of $I$ is unramified at all finite primes different from $p$ and $K^{I} / \mathbb{Q}$ has nilpotent Galois group. By Lemma 9, there is a unique prime in $K^{I}$ over $p$. Theorem 1 applied to $G(p) \subseteq \mathrm{GL}_{n}\left(K^{I}\right)$ implies that $G(p)$ can be conjugated to diagonal matrices by an element in $\mathrm{GL}_{n}(\mathbb{Z})$. In particular, the group $G(p)$ is a commutative $p$-group for every prime $p$. If $p \neq q$ then clearly the groups $G(p)$ and $G(q)$ commute.

An easy inductive argument shows that the whole group $D$ generated by the subgroups $G(q)$ can be conjugated to diagonal matrices by an element of $\mathrm{GL}_{n}(\mathbb{Z})$. In fact, $D$ is a commutative abelian group with $p$-Sylow subgroup $G(p)$. Fix a prime $p$ such that $G(p)$ is not trivial and let $D(p)$ be the product of all Sylow subgroups of $D$ different from $G(p)$. Theorem 1 allows us to assume that $G(p)$ is in a strongly diagonal form. Clearly $D(p)$ centralizes $G(p)$ and therefore it is contained in the centralizer of $G(p)$ in $\mathrm{GL}_{n}$. As noted in Section 2, this centralizer consists of block-diagonal matrices, i.e. it is a product of $\mathrm{GL}_{m}$ 's, and in each of these, the elements of $G(p)$ are scalar matrices. Thus we can use induction and conjugate $D(p)$ to diagonal matrices by an element in $\mathrm{GL}_{n}(\mathbb{Z})$ which centralizes $G(p)$. Hence we can assume that $D$ is in a strongly diagonal form. Clearly $D$ is normal in $G$. Note that $\Gamma$ acts trivially on $G / D$. In fact, for any prime $\beta$ of $K$ the inertia $I(\beta)$ acts trivially on $G / G(\beta)$ so it acts trivially on $G / D$. Since all the inertia groups generate $\Gamma$ (because $\mathbb{Q}$ has no unramified extensions), our claim follows.

By our discussion of the normalizer of $D$ in Section 2 it follows that any element $g$ of $G$ is of the form $g=c w$, where $c$ centralizes $D$ and $w$ is a permutation matrix in $\Pi_{D}$. Plainly $c \in \mathrm{GL}_{n}\left(O_{K}\right)$. Since $\Gamma$ acts trivially on $G / D$, for any $\tau \in \Gamma$ there is $d \in D$ such that $g^{\tau} g^{-1}=c^{\tau} c^{-1}=d$. Now the centralizer of $D$ in $\mathrm{GL}_{n}$ is a product of $\mathrm{GL}_{m}$ 's and if we take a component $C$ of $c$ in one of these $\mathrm{GL}_{m}$ 's then we have $C^{\tau}=C \xi$ where $\xi$ is a root of unity (corresponding component of $d$ ). By Lemma 8 we get $C=\zeta M$, where $M$ is an invertible integral matrix and $\zeta$ is a root of 1 . Thus $c=b m$ where $b$ is a diagonal matrix of finite order and $m \in \mathrm{GL}_{n}(\mathbb{Z})$ centralizes $D$ and $b$. Thus we proved that any element of $G$ is of the form $b m w$ where $b$ is diagonal of finite order, $m \in \mathrm{GL}_{n}(\mathbb{Z})$ centralizes $b$ and $D$, and $w$ is a permutation matrix in $\Pi_{D}$. This is exactly what we need in order to say that $G$ is of A-type. 


\section{References}

[1] Y. Kitaoka, Arithmetic of Quadratic Forms, Cambridge Tracts in Math. 106, Cambridge Univ. Press, 1993.

[2] Y. Kitaoka and H. Suzuki, Finite arithmetic subgroups of $G L_{n}, I V$, Nagoya Math. J. 142 (1996), 183-188.

[3] M. Mazur, Finite arithmetic subgroups of $G L_{n}$, J. Number Theory 75 (1999), 109-119.

[4] - Finite arithmetic subgroups of $G L_{n}$, part 1 of the University of Chicago PhD. thesis, 1999.

[5] - Finite flat group schemes and a conjecture of Kitaoka, in preparation.

[6] V. P. Platonov and A. S. Rapinchuk, Algebraic Groups and Number Theory, Pure Appl. Math. 139, Academic Press, Boston, MA, 1994.

Department of Mathematics

University of Illinois at Urbana-Champaign

1409 W. Green Street

Urbana, IL 61801-2975

E-mail: mazur1@math.uiuc.edu

Received on 28.2.2000

and in revised form on 17.11.2000 